\title{
Application of disturbance observer-based control in low-level radio-frequency system in a compact energy recovery linac at KEK
}

\author{
Feng Qiu, 1,2,* Shinichiro Michizono, ${ }^{1,2}$ Takako Miura, ${ }^{1,2}$ Toshihiro Matsumoto,,2 \\ Mathieu Omet, ${ }^{2}$ and Basuki Wibowo Sigit $^{2}$ \\ ${ }^{1}$ High Energy Accelerator Research Organization, 1-1 Oho, Tsukuba, Ibaraki 305-0801, Japan \\ ${ }^{2}$ The Graduate University for Advanced Studies, Shonan Village, Hayama, Kanagawa 240-0193, Japan
}

(Received 24 May 2015; published 28 September 2015)

A disturbance observer (DOB)-based control for a digital low-level radio-frequency (LLRF) system in a compact energy recovery linac (cERL) at KEK has been developed. The motivation for this control approach is to compensate for or suppress the disturbance signal in the rf system such as beam loading, power supply ripples, and microphonics. Disturbance signals in specified frequency ranges were observed and reconstructed accurately in the field-programmable gate array and were then removed in the feedforward model in real time. The key component in this DOB controller is a disturbance observer, which includes the inverse mathematical model of the rf plant. In this paper, we have designed a DOB control-based approach in order to improve the LLRF system performance in disturbance rejection. We have confirmed this approach in the cERL beam commissioning.

DOI: 10.1103/PhysRevSTAB.18.092801

PACS numbers: 29.20.-c, 29.27.-a

\section{INTRODUCTION}

In KEK, a compact energy recovery linac (cERL), which is a test facility for future $3 \mathrm{GeV}$ ERL accelerators, was constructed $[1,2]$. The cERL is a $1.3 \mathrm{GHz}$ superconducting radio-frequency (SCRF) machine that is operated in continuous-wave (cw) mode [3]. A total of three two-cell cavities were installed in the injector, and two nine-cell cavities were installed in the main linac $[4,5]$. The layout of the cavities and the rf source is illustrated in Fig. 1. In order to fulfill the desired beam quality requirements, the rf field fluctuation should be maintained at less than $0.1 \%$ for the amplitude and $0.1^{\circ}$ for the phase. For future $3 \mathrm{GeV}$ ERL, these requirements are even tighter $(0.01 \%$ for the amplitude and $0.01^{\circ}$ for the phase). A field-programmable gate array (FPGA)-based digital low-level radio-frequency (LLRF) system has been employed to regulate and control the rf field [6].

In a LLRF system, disturbances such as microphonics, power supply ripples, and beam loading will severely limit the system performance. For instance, in the LLRF systems of the cERL, main disturbances include the $300 \mathrm{~Hz}$ highvoltage power supply (HVPS) ripples in the two-cell cavities of the injector and the microphonics (with dominant $49.6 \mathrm{~Hz}$ ) in the nine-cell cavities of the main linac $[6,7]$. Furthermore, during the beam commissioning, approximately $1.6 \mathrm{~ms}$ and $1 \mathrm{~mA}$ beam current in burst

\footnotetext{
*qufeng@ post.kek.jp

Published by the American Physical Society under the terms of the Creative Commons Attribution 3.0 License. Further distribution of this work must maintain attribution to the author(s) and the published article's title, journal citation, and DOI.
}

mode is operated. In principle, the beam current under burst mode is repetitive and predictable, therefore, the beamloading profile can be prestored and then removed from the feedforward (FF) model. However, at cERL LLRF systems, the FF model inside the FPGA is simplified as a single register, which means, a prestored array (table) for beam compensation is not available actually. Thus, the beam loading is another disturbance that needs to be considered at cERL $[2,8]$. Table I summarizes the main disturbances of each cavity at cERL.

In principle, the disturbances can be rejected or compensated for by applying higher feedback (FB) gains. However, owing to the existence of the loop delay, the FB gains cannot be increased beyond certain limits. In view of this situation, a disturbance observer-based (DOB) control is a promising approach for suppressing the disturbances [9-20]. This approach was first introduced by Ohnishi [9] and refined by Umeno and Hori [11]. The disturbances in the specified frequency range (e.g., DC to $5 \mathrm{kHz}$ ) are observed and regenerated by the DOB controller and are then subtracted from the FF model. Because the disturbance signal is normally concentrated in the lowfrequency bands (less than $5 \mathrm{kHz}$ ) in the cERL LLRF system and because prior knowledge of the system model is known to some extent, the disturbance signal can be estimated and then removed reliably. Therefore, the restriction of high FB gain control can be relaxed. In the cERL beam commissioning, we have successfully suppressed disturbances such as the $300 \mathrm{~Hz}$ power supply ripples, the $1 \mathrm{~mA}, 1.6 \mathrm{~ms}$ beam loading, and the microphonics by applying this proposed DOB approach.

This paper focuses on the principle, design, and implementation of the DOB control approach mentioned above. 


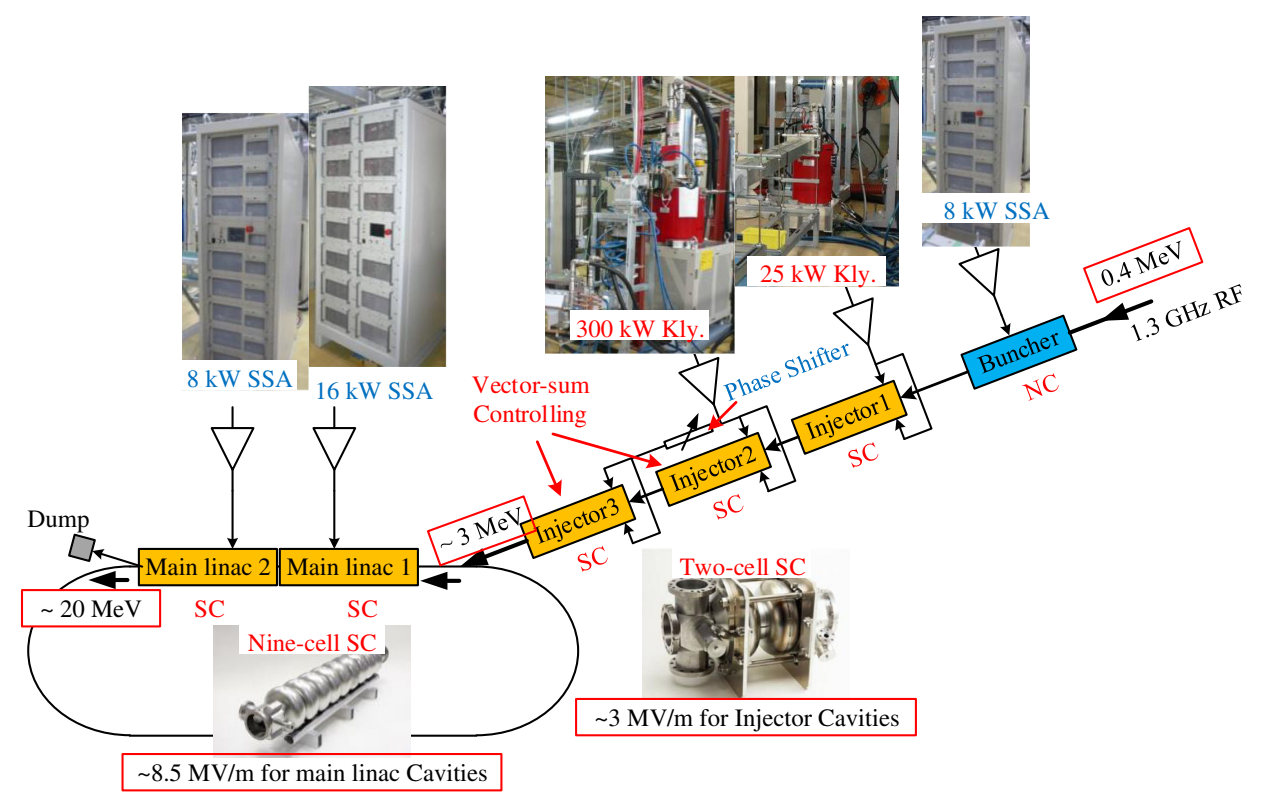

FIG. 1. Layout of the cERL. The cavities, rf source, and beam energy are presented in the figure. The marked values of the beam energy and electric field indicate the current state during beam commissioning.

The improvement of this DOB-based application at cERL in terms of regulation performance is presented as well. The organization of this paper is as follows. Section II briefly introduces the structure of the LLRF in the cERL. Section III describes the principle and structure of the DOB control. Section IV depicts the FPGA implementation of the DOB control. Section V presents the analytical method and some simulation results of the controller with Matlab. Section VI presents the results of that controller in cERL commissioning and the improvement of the controller as compared to traditional proportional and integrational (PI) controls [21]. In addition, Sec. VI also summarizes the limitations of this method. Finally, Sec. VII summarize this DOB control-based approach.

\section{LLRF SYSTEM}

A simplified block diagram of the cERL LLRF system is shown in Fig. 2. The $1.3 \mathrm{GHz}$ rf signal (from cavity probe) is down-converted to a $10 \mathrm{MHz}$ intermediate frequency (IF) signal. The IF signal is sampled in the next stage at $80 \mathrm{MHz}$ by a 16-bit analog to digital converter (ADC, LTC2208)

TABLE I. Main disturbances of the superconducting (SC) cavities at cERL.

\begin{tabular}{ll}
\hline \hline Cavity & \multicolumn{1}{c}{ Main disturbance } \\
\hline Injector 1 & Beam loading and microphonics \\
Injector 2 & Beam loading and the 300 Hz HVPS ripples \\
Injector 3 & Beam loading and the 300 Hz HVPS ripples \\
Main linac 1 & Microphonics \\
Main linac 2 & Microphonics (dominant 49.6 Hz) \\
\hline \hline
\end{tabular}

and fed into a field-programmable gate array (FPGA). The base-band in phase and quadrature (I/Q) components are extracted from the digitalized IF signal by a digital I/Q detection model [22]. The notation "I/Q" derives from fact that any sinusoidal signal can be represented by either polar (amplitude/phase) or by Cartesian coordinates. A sinusoidal signal $y(t)=A \sin \left(\omega t+\phi_{0}\right)$ with amplitude $A$, radian frequency $\omega$, and an initial phase $\phi_{0}$, can be decomposed into its sin and cos component by basic trigonometric functions [23]:

$$
\begin{aligned}
y(t) & =A \sin \left(\omega t+\phi_{0}\right) \\
& =\underbrace{A \cos \phi_{0}}_{I} \sin (\omega t)+\underbrace{A \sin \phi_{0}}_{Q} \cos (\omega t) .
\end{aligned}
$$

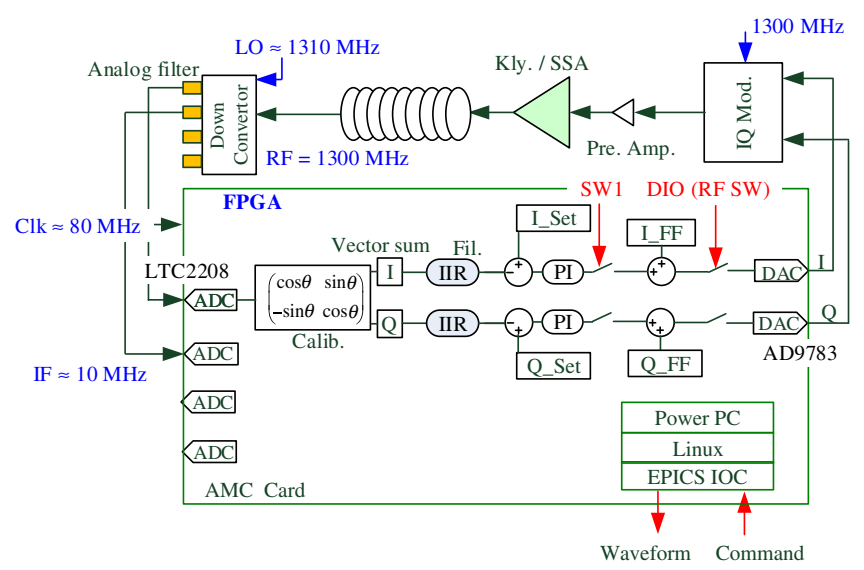

FIG. 2. LLRF system diagram in the cERL at KEK. SW1 marked in the figure provides the hand-off between the FF and FB operations. 
The amplitude of the sin component and the cos component is then defined as the in-phase component (I) and the quadrature-phase component (Q). The I/Q signals are fed into a $2 \times 2$ rotation matrix to correct the loop phase. After being filtered by infinite impulse response (IIR) low-pass filters, the I/Q components are compared to their set values and the I/Q errors are calculated. Then, the I/Q errors are regulated by a proportional-integral (PI) controller. The processed I/Q signals are added to their corresponding FF models. The combinational signals are fed into the I/Q modulator by a 16-bit digital to analog converter (DAC, AD9783) to modulate the $1.3 \mathrm{GHz}$ rf signal from the oscillator. Finally, the LLRF feedback loop is closed by driving a high-power source, which drives the cavities.

There are two basic operation modes in the LLRF system: the FF operation and the FB operation. In an FF operation, the FF model directly drives the high-power source without responding to how the cavity reacts. In contrast, in the FB operation, the high-power source is driven only by the PI controller output without activating the FF model. In the cERL, the FF operation is mainly used to preliminarily establish a rough rf field in a cavity, whereas the FB operation takes charge of high-precision field control. The FF/FB operation is denoted as SW1 in Fig. 2.

A typical rf system is subjected to various disturbances. Typical sources include known disturbances such as HVPS ripples, Lorentz detuning and beam loading, and unknown disturbances such as microphonics and master oscillator phase noise. To achieve the best performance, structures and parameters of the FB controller need to be carefully selected, e.g., the PI gain needs to be optimized by a gainscanning experiment [24-27]. Generally speaking, an optimized PI controller works well for rejecting the lowfrequency disturbances such as the microphonics at $10 \mathrm{~Hz}$. However, in the presence of higher frequencies and largerintensity disturbances (e.g., $300 \mathrm{~Hz}$ PS ripples and $1.6 \mathrm{~ms}$ beam currents), even an optimized PI controller is usually not sufficient. This paper describes our experience with applying a successful solution to disturbance rejection problems known as "disturbance observer-based (DOB) controls."

\section{DOB CONTROL}

The basic architecture of DOB control is illustrated in Fig. 3(a) $[9,10,12,17]$. Signals $u, \varepsilon, d$, and $\hat{d}$ represent the command, plant input, disturbance, and disturbance estimate, respectively. Models $G_{p}(s)$ and $G_{n}(s)$ represent the transfer function of the real plant (combination of cavity, rf sources, preamplifier, I/Q detector, etc.) and nominal plant model. According to Fig. 3(a), the disturbance estimation is given by [17]

$$
\hat{d}=(\varepsilon+d) G_{p}(s) G_{n}^{-1}(s)-\varepsilon .
$$

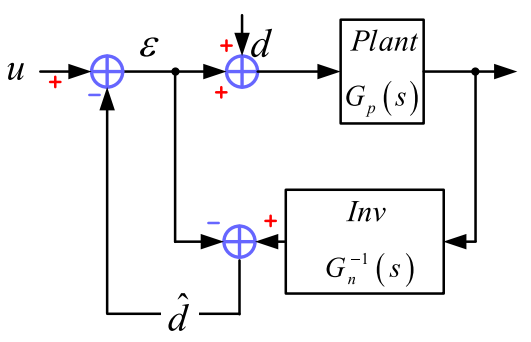

(a)

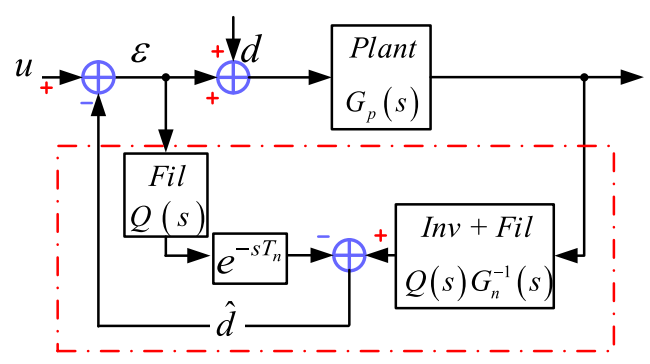

(b)

FIG. 3. Architecture of DOB control: (a) the basic structure of DOB control, (b) DOB control with $Q$ filter and time-delay compensation unit.

Therefore, if the nominal plant model is accurate enough, $G_{p}(s)$ in (2) will be canceled out by $G_{n}^{-1}(s)$. As a result, the disturbance signal $d$ can be reproduced by $\hat{d}$. After removing $\hat{d}$ from the control signal $u$, the disturbance signal $d$ would be compensated by $\hat{d}$. That is the basic idea of DOB control.

\section{A. Principle of DOB controller}

Usually, the relative degree (the difference between the order of the denominator and the order of the numerator in a system) of $G_{p}(s)$ is greater than zero. This leads to an anticasual inverse model, which is not physically realizable. This problem can be resolved by connecting $G_{n}^{-1}(s)$ with a low-pass $Q(s)$ filter, as shown in Fig. 3(b) [10,17-19]. To make the overall combination $Q(s) G_{n}^{-1}(s)$ remain casual, the relative degrees of the $Q$ filter must be greater than or at least equal to that of $G_{n}(s)$. The determination of the $Q$ filter is a compromise between performance, robustness, and complexity. A good candidate is given by $[11,12,19,20]$

$$
Q_{n m}(s)=\frac{\sum_{k=0}^{n} \alpha_{k} \cdot(\tau s)^{k}}{(\tau s+1)^{m}}, \quad \alpha_{k}=\frac{m !}{(m-k) ! k !},
$$

where $\tau$ is the filter time constant, $\alpha_{k}=\frac{m !}{(m-k) ! k !}$ is the binomial coefficient, and $m$ and $n$ are the order of the denominator and numerator, respectively. The low-pass $Q$ filter is significant because it not only restricts the effective bandwidth of the DOB controller, but also determines the 
robust stability of the system [17]. The detailed discussion of the $Q$ filter will be presented in a following paper (see Sec. V).

Another issue which needs to be considered is the time delay. The actual rf plant has the time delay, $T_{d}$, and it should be also considered in the real application. Figure 3(b) provides the architecture of the DOB control in an actual system $[15,17,20]$, the DOB controller is indicated by the red block. The $Q(s)$ in Fig. 3(b) is the low-pass $Q$ filter mentioned above, and the nominal time delay $T_{n}$ is used to compensate the actual plant delay, $T_{d}$. In a following paper, we will discuss each component in detail.

\section{B. Design of DOB controller}

From the discussion above, the nominal system model (open-loop models from FF to IIR filter output in Fig. 2 under FF operation) $G_{n}(s)$ is required in the DOB control. This model can be acquired by a modern system identification method [28]. Generally, the identified model of the LLRF system is a multi-input multi-output (MIMO) system. For example, a classic mode of an LLRF system in transfer function form is given by a $2 \times 2$ transfer function matrix:

$$
G_{n}(s)=\left(\begin{array}{ll}
G_{11}(s) & G_{12}(s) \\
G_{21}(s) & G_{22}(s)
\end{array}\right),
$$

with transfer functions

$$
G_{i j}(s)=\frac{b_{i j, m} s^{m}+b_{i j, m-1} s^{m-1}+\cdots+b_{i j, 0}}{s^{n}+a_{i j, n-1} s^{n-1}+\cdots+a_{i j, 0}},
$$

where the orders $m$ and $n$ are determined by an identification algorithm.

The inverse transfer function of $G_{n}(s)$ given by (4) and (5) can be calculated by MATLAB. In principle, the $G_{n}^{-1}(s)$ is also MIMO structure as shown in (4) and (5). However, in the cERL, the Lorentz force detuning effect is not serious due to the cw mode operation, and the detuning is well controlled by the piezo tuner $[6,29]$. That is to say, the cavity is worked almost in the on-resonance case and the cross-coupling components of (4), $G_{12}(s)$ and $G_{21}(s)$, can be neglected.

Furthermore, the cERL project has adopted superconducting cavities that are operated in cw mode. The loaded Q value $\left(Q_{L}\right)$ of the cavities is usually rather high (e.g., the loaded Q of the cavities in the main linac is more than $10^{7}$ ), which means, the corresponding cavity half-bandwidth, $f_{0.5}$, is very low according to

$$
f_{0.5}=\frac{f_{0}}{2 Q_{L}},
$$

where $f_{0}$ is the resonance frequency of the superconducting cavity $\left(f_{0}=1.3 \mathrm{GHz}\right.$ in cERL). Generally, the bandwidth of a cavity is much smaller than other components such as the I/Q modulator, the power source, and I/Q detector. In this case, the only dominant poles of the open-loop model are contributed by the superconducting cavity, i.e., in the diagonal components of (4). The transfer functions, $G_{11}(s)$ and $G_{22}(s)$, can be then simplified by

$$
G_{11}(s)=G_{22}(s)=G_{f f} \cdot \frac{2 \pi f_{0.5}}{s+2 \pi f_{0.5}},
$$

where the parameter $G_{f f}$ is the plant gain in the LLRF system (amplitude gain from FF to IIR filter output at steady state in Fig. 2 under the FF operation).

Therefore, the previous $2 \times 2$ matrix in (4) is simplified to

$$
G_{n}(s)=G_{f f} \cdot\left(\begin{array}{cc}
\frac{2 \pi f_{0.5}}{s+2 \pi f_{0.5}} & 0 \\
0 & \frac{2 \pi f_{0.5}}{s+2 \pi f_{0.5}}
\end{array}\right) .
$$

Correspondingly, the inverse transfer function, $G_{n}^{-1}(s)$, is then given by

$$
G_{n}^{-1}(s)=\frac{1}{G_{f f}}\left(\begin{array}{cc}
\frac{s+2 \pi f_{0.5}}{2 \pi f_{0.5}} & 0 \\
0 & \frac{s+2 \pi f_{0.5}}{2 \pi f_{0.5}}
\end{array}\right) .
$$

It is clear to see from (8) and (9) that the only two parameters we need to identify are the cavity half-bandwidth, $f_{0.5}$, and the plant gain, $G_{f f}$. According to analytical study and the experience in the beam commissioning, the robustness of the DOB controller is strong enough, that is, even rough identification of the system model achieves good system performance. Since the system halfbandwidth, $f_{0.5}$, can be calculated by (6) directly (the loaded $Q$ value is usually known in advance), the plant gain can be easily calibrated as well. This means that we can design the DOB controller even without performing the system identification experiment.

The $Q$ filter indicated by (3) and Fig. 3(b) is another key point in our controller design. As discussed above, the combination of the $Q$ filter and the nominal model, $G_{n}(s)$, should be casual. Therefore, according to the expression in (7), the relative degree of $Q(s)$ should be greater than or equal to 1 (that is, $m-n \geq 1$ ). The values of $n$ and $m$ should not be too large, otherwise the cost of the FPGA implementation becomes too high. The final form of the $Q$ filter is given by a $2 \times 2$ matrix:

$$
Q(s)=\left(\begin{array}{cc}
Q_{n m}(s) & 0 \\
0 & Q_{n m}(s)
\end{array}\right) .
$$

The nominal plant delay, $T_{n}$, is identified by exciting the LLRF system with a square wave in the DAC output [26] under FF operation, and the measured plant delay is 


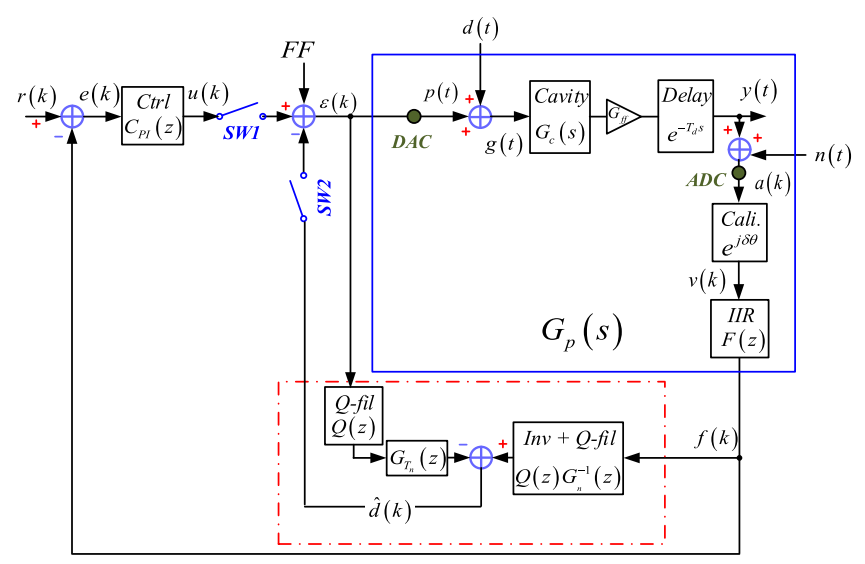

FIG. 4. Overall model of "PI + DOB" control. The presented DOB controller is indicated by the red block. Detailed definitions of each parameter are given in Table II.

approximately $1 \mu$ s according to our experiment. Because the frequency of the interested disturbance signal is less than $5 \mathrm{kHz}$, the effects of this $1 \mu$ s delay are not serious. In our study, we just selected $T_{n}=1 \mu \mathrm{s}$ as our nominal plant delay.

TABLE II. Definition of the parameters and models in Fig. 4.

\begin{tabular}{|c|c|}
\hline Parameters & Definition \\
\hline$y(t)$ & Cavity pickup signal \\
\hline$p(t)$ & DAC output signal \\
\hline$d(t)$ & Disturbance signal \\
\hline$g(t)$ & Cavity input signal \\
\hline$n(t)$ & Measurement noise signal \\
\hline$r(k)$ & Reference signal \\
\hline$\hat{d}(k)$ & Disturbance estimate signal \\
\hline$a(k)$ & Digital cavity pickup signal (ADC output) \\
\hline$v(k)$ & Phase calibration model output \\
\hline$f(k)$ & IIR filter output \\
\hline$e(k)$ & Difference between $r(k)$ and $f(k)$ \\
\hline$u(k)$ & PI controller output \\
\hline$\varepsilon(k)$ & DAC input \\
\hline$F F$ & FF model (a single value at cERL) \\
\hline$G_{f f}$ & Gain from $F F$ to $f(k)$ at steady state \\
\hline$\delta \theta$ & Residual phase calibration error \\
\hline$e^{j \delta \theta}$ & Phase rotation model \\
\hline$G_{c}(s)$ & SC cavity model \\
\hline$e^{-T_{d}}(s)$ & Actual plant delay model \\
\hline$C_{\mathrm{PI}}(z)$ & PI controller \\
\hline$F(z)$ & IIR filter \\
\hline$Q(z)$ & $Q$ filter \\
\hline$G_{n}(z)$ & Simplified nominal plant model [see Eq. (8)] \\
\hline$G_{T_{n}}(z)$ & Nominal model of plant delay \\
\hline$G_{p}(s)$ & Plant model $\left(G_{p}=G_{c} G_{f f} e^{-T_{d} s} e^{j \delta \theta} F\right)$ \\
\hline
\end{tabular}

Up to now, the components of the DOB controller have been described in detail. Finally, we will integrate the DOB controller shown in Fig. 3(c) with the PI controller. Figure 4 shows the combination of DOB control and PI control. The outputs of the DOB controller are connected to the FF model directly to assist the PI control. To distinguish the continuous time domain and discrete time domain of an actual digital LLRF system, we use the notation " $t$ " and " $s$ " for the former case, and " $k$ " and " $z$ " for the latter case.

The definition of each parameter and model is summarized in Table II. The IIR filter, $F(z)$, is used to suppress the high-frequency noise, $n$ (e.g., ADC clock jitter, white noise) and other resonance modes of cavities [25]. Generally, the bandwidth of $F(z)$ is far larger than $G_{p}(z)$ and $Q(z)$. Usually, we need to calibrate the loop phase of the LLRF system, and in principle, the loop phase can be compensated by the phase rotation matrix in Fig. 2, however, residual error of the loop phase still exists in the system. Therefore, we add an rotation model $e^{j \delta \theta}$ to simulate this case in Fig. 4. The $\delta \theta$ here indicate the residual phase calibration error. The model $G_{T_{n}}(z)$ here is the discrete form of the nominal delay model in Fig. 3. The switch "SW2" engages the enable/disable operation of the DOB controller (indicated by the red block in Fig. 4), whereas "SW1" takes charge of the FF/FB operation.

\section{IMPLEMENTATION OF DOB CONTROLLER IN FPGA}

In order to implement the DOB controller in FPGA, we have to convert the DOB controller in continuous-time form to its discrete-time form. We will define our method for the continuous-time to discrete-time conversion in detail.

The digital form of the simplified nominal rf model, $G_{n}(s)$, in (8) is given by

$$
G_{n}(z)=G_{f f} \cdot\left(\begin{array}{cc}
\frac{(1-\beta) z^{-1}}{1-\beta z^{-1}} & 0 \\
0 & \frac{(1-\beta) z^{-1}}{1-\beta z^{-1}}
\end{array}\right)
$$

Here, we match the pole in (8) and (11). Since the pole in (8) is $s_{p}=-2 \pi f_{0.5}$ in $G_{n}(s)$, the pole $z_{p}=\beta$ in (11) can be defined by

$$
z_{p}=\beta=e^{s_{p} T_{s}}=e^{-2 \pi f_{0.5} T_{s}}
$$

where the parameter $T_{s}$ is a sampling period of the FPGA board in the LLRF system. The difference equation of $G_{n}^{-1}(z)$ inside FPGA is then expressed as

$$
y(k-1)=\frac{x(k)-\beta x(k-1)}{(1-\beta) G_{f f}},
$$


where the sequence $x(k)$ and $y(k)$ represents the input sequence and output sequence of $G_{n}^{-1}(z)$ inside FPGA.

The $Q$ filter in discrete-time form, $Q(z)$, is transformed using the pole matching method similar with (11):

$$
Q(z)=\left(\begin{array}{cc}
\frac{(1-\gamma)^{2} z^{-2}}{\left(1-\gamma z^{-1}\right)^{2}} & 0 \\
0 & \frac{(1-\gamma)^{2} z^{-2}}{\left(1-\gamma z^{-1}\right)^{2}}
\end{array}\right) .
$$

We used digital delay model $G_{T_{n}}(z)$ to compensate for the plant delay $T_{d}$ :

$$
G_{T_{n}}(z)=z^{-L}, \quad \text { and } \quad L=\left\lceil\frac{T_{n}}{T_{s}}\right\rceil,
$$

where the operator " \lceil\rceil " means round the element inside the brackets to the nearest integers towards 0 .

\section{ANALYTICAL STUDY OF DOB CONTROL}

An analytical study of the DOB control shown in Fig. 4 was carried out by utilizing the Bode plots and $H_{\infty}$ norm. The SC cavity model $G_{c}(s)$ is selected to be the classic cavity baseband state differential equation [30] on the cavity of injector $1\left(Q_{L}=1.2 \times 10^{7}\right)$ :

$$
\begin{aligned}
\frac{d}{d t}\left(\begin{array}{c}
V_{I} \\
V_{Q}
\end{array}\right)= & \left(\begin{array}{cc}
-\omega_{0.5} & -\delta \omega \\
\delta \omega & -\omega_{0.5}
\end{array}\right)\left(\begin{array}{c}
V_{I} \\
V_{Q}
\end{array}\right) \\
& +\left(\begin{array}{cc}
\omega_{0.5} R_{L} & 0 \\
0 & \omega_{0.5} R_{L}
\end{array}\right)\left(\begin{array}{c}
I_{I} \\
I_{Q}
\end{array}\right),
\end{aligned}
$$

where the parameters $\left(V_{I}, V_{Q}\right)$ and $\left(I_{I}, I_{Q}\right)$ are the I/Q components of the cavity voltage, and drive current, respectively. The parameters $\omega_{0.5}$ and $\delta \omega$ represent the radian cavity half-bandwidth and detuning, respectively. $R_{L}$ is the load resistance. In the on-resonance case $(\delta \omega=0)$, the cavity transfer function is

$$
G_{c}(s)=\left(\begin{array}{cc}
\frac{\omega_{0.5}}{s+\omega_{0.5}} & 0 \\
0 & \frac{\omega_{0.5}}{s+\omega_{0.5}}
\end{array}\right) .
$$

For the analytical study, first of all, we consider the simplest case. We assume the cavity is on resonance $(\delta \omega=0)$, and the loop phase is perfectly calibrated $(\delta \theta=0$ in Fig. 4). In this case, there are no cross components in the system, thus the overall LLRF system is actually a single input single output (SISO) system. The $Q$ filter here is a $5 \mathrm{kHz}$ second order low-pass filter which will be discussed in detail in the following paper (see Table III). The gains in the PI controller are determined according to the gain-scanning result in [24-27]. Besides, we suppose that there is a $1 \mu \mathrm{s}$ plant delay $T_{d}$ and $T_{n}$. Figure 5 plots the bode diagrams of the main models. The
TABLE III. Transfer function of the $Q_{n m}$ filters.

\begin{tabular}{lc}
\hline$Q$ filter & Transfer function \\
\hline$Q_{01}(s)$ & $\frac{1}{\tau s+1}$ \\
$Q_{02}(s)$ & $\frac{1}{(\tau s+1)^{2}}$ \\
$Q_{13}(s)$ & $\frac{3 \tau s+1}{(\tau s+1)^{3}}$ \\
\hline \hline
\end{tabular}

analytical study of this paper is carried out based on the models given in Fig. 5.

\section{A. Fundamental analysis}

If we operate the system with both PI control and DOB control (switch on both SW1 and SW2 in Fig. 4), then according to Mason's rule [31], the characteristic polynomial $\Delta_{\text {tol }}$ (system determinant) in the overall schematic is defined by

$$
\Delta_{\mathrm{tol}}=1-(\underbrace{Q G_{T_{n}}}_{L_{1}} \underbrace{-G_{p} Q G_{n}^{-1}}_{L_{2}} \underbrace{-C_{P I} G_{p}}_{L_{3}}),
$$

where the parameters $L_{1}, L_{2}$, and $L_{3}$ represent the internal loops in Fig. 4.

If we operate the system with DOB control but without PI control (switch on SW2 and switch off SW1), the characteristic polynomial $\Delta_{\mathrm{DOB}}$ is then given by

$$
\Delta_{\mathrm{DOB}}=1-(\underbrace{Q G_{T_{n}}}_{L_{1}} \underbrace{-G_{p} Q G_{n}^{-1}}_{L_{2}}) .
$$
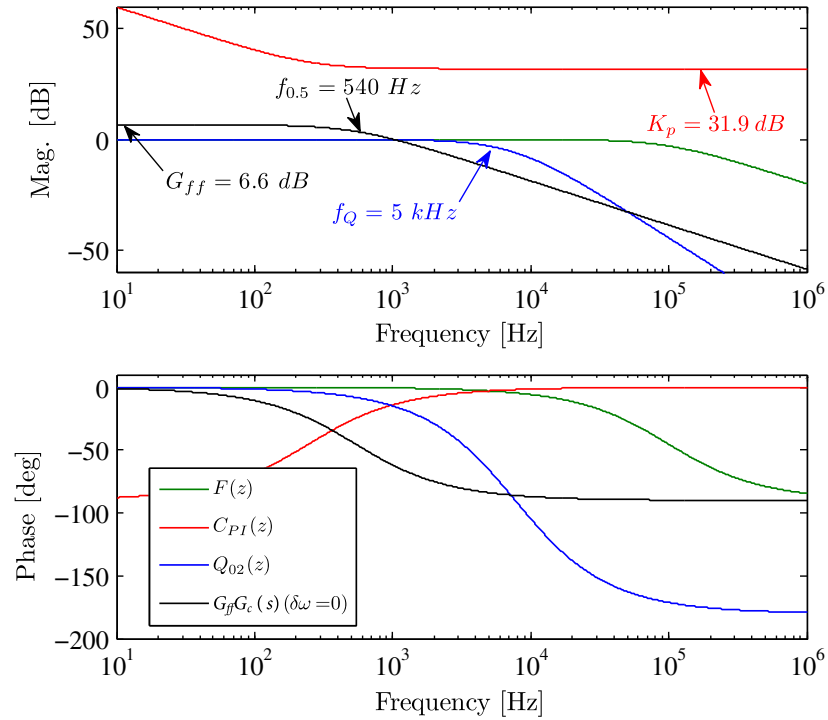

FIG. 5. Bode plots of the $F(z), C_{P I}(z), Q_{02}(z)$ and $G_{f f} G_{c}(s)$. The models applied in the analytical study in this paper are mainly based on this figure. 
Finally, if we operate the system with PI control only (switch on SW1 and switch off SW2), the characteristic polynomial $\Delta_{\mathrm{PI}}$ becomes

$$
\Delta_{\mathrm{PI}}=1-(\underbrace{-C_{\mathrm{PI}} G_{p}}_{L_{3}}) .
$$

The open-loop bode plots of these three cases are illustrated in Fig. 6. Here, we suppose the cavity is on resonance $(\delta \omega=0)$, in this case, the poles in cavity model $G_{c}$ are perfectly canceled by the zeros in the simplified inverse nominal plant model, $G_{n}^{-1}$. Furthermore, suppose the plant delay $T_{d}$ is perfectly compensated by the nominal delay $T_{n}$ and there is no phase calibration error in the system $(\delta \theta=0)$, under these conditions discussed above, the characteristic polynomial $\Delta_{\text {tol }}$ then becomes

$$
\begin{aligned}
\Delta_{\mathrm{tol} 0} & =1-\left(Q G_{T_{n}}-e^{-T_{d} s} F Q-C_{\mathrm{PI}} G_{p}\right) \\
& =1-\left[e^{-T_{d} s} Q(1-F)-C_{\mathrm{PI}} G_{p}\right] .
\end{aligned}
$$

In practice, the bandwidth of the low-pass IIR filter $F(z)$ is much more larger than the $Q$ filter, which means, the equation $F(z) \approx 1$ is satisfied in the interested frequency ranges (e.g., DC to $50 \mathrm{kHz}$ ). With this condition, we have $\Delta_{\text {tol0 }} \approx \Delta_{\text {PI }}$. As shown in Fig. 6 , the open-loop bode plots of the PI + DOB control and PI control almost overlap, which means that the introduction of the DOB structure will not influence the total characteristic polynomial. In addition, in the case of the DOB individual control such as (19), it is easy to see from the open-loop plots (indicated by the blue dash-dotted line) that the margins are infinite owing to the fact that the magnitude gain is always less than $0 \mathrm{~dB}$.

Figure 7 compares several kinds of closed-loop system responses of these three controllers. Similar with Fig. 6, we suppose that the block $G_{c} G_{f f}$ is perfectly canceled out by $G_{n}^{-1}$. It should be emphasized that the values of the PI gains
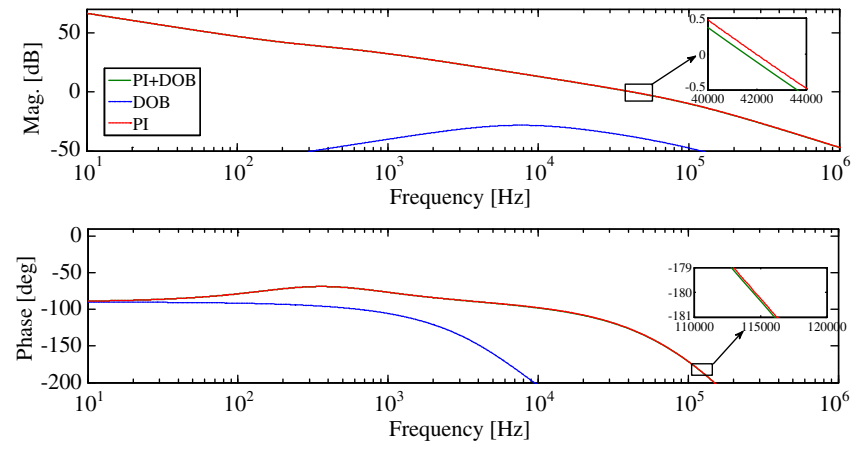

FIG. 6. Open-loop comparison of the three cases described above (assuming $\delta \omega=0$ and $\delta \theta=0$ ). The curves in the PI + DOB case (indicated by the green solid line) and the PI case (indicated by the red dashed line) almost overlap. The DOB individual control is plotted by the blue dash-dotted line.

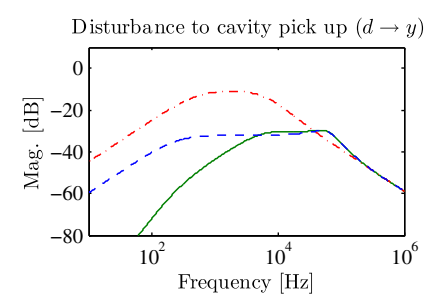

(a)

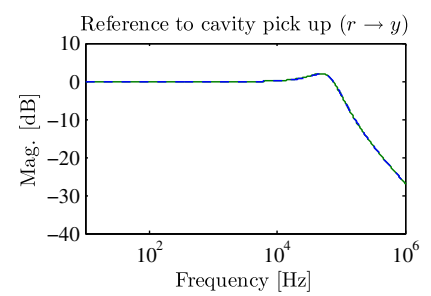

(c)

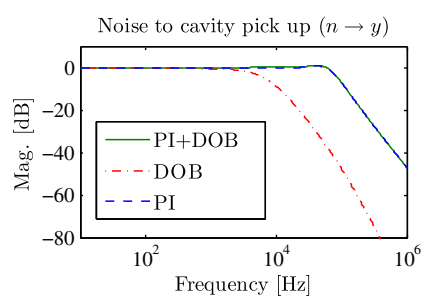

(b)

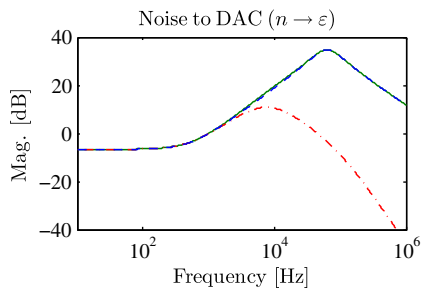

(d)
FIG. 7. Comparison of the closed-loop system response of the three controls (assuming $\delta \omega=0$ and $\delta \theta=0$ ). Here, we select $Q_{02}(s)$ with $5 \mathrm{kHz}$ bandwidth as the $Q$ filter (see Table III). (a) Disturbance to cavity pickup ( $\rightarrow \rightarrow y$ ), (b) Noise to cavity pickup $(n \rightarrow y)$. (c) Reference to cavity pickup $(r \rightarrow y)$. (d) Noise to DAC $(n \rightarrow \varepsilon)$. The interpretation and location of the signal shown in Fig. 7 are presented in Table II and Fig. 4, respectively.

in both the PI + DOB control and the PI control were selected based on the same optimal value of the injector 1 cavity, [24-27]. The interpretation and location of the signals shown in Fig. 7 are given in Table II and Fig. 4, respectively.

Figure 7(a) plots the transfer function from the disturbance signal, $d$, to the cavity pickup signal, $y$. The results indicate that the presented PI + DOB structure shows great superiority in terms of disturbance rejection for the frequency range from DC to about $5 \mathrm{kHz}$. This characteristic is also the most attractive advantage of the DOB control.

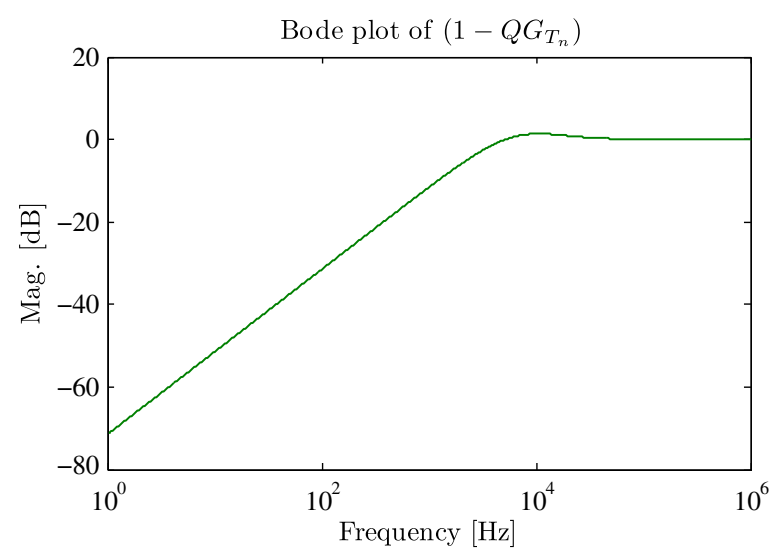

FIG. 8. Bode plots of the block, $1-Q G_{T_{n}}$. The magnitude response indicated that the inequalities $1-Q G_{T_{n}}<0 d B$ is satisfied in the frequency range from DC to $5 \mathrm{kHz}$, as a result, the response from $d$ to $y$ in the $\mathrm{PI}+\mathrm{DOB}$ control will be improved in the corresponding frequency ranges. 
The improvement of the PI + DOB control can be explained by its closed loop transfer function. If the cavity is on resonance, the transfer function of $\mathrm{PI}+\mathrm{DOB}$ is given by

$$
H_{\mathrm{tol} 0, d \rightarrow y}=\frac{\left(1-Q G_{T_{n}}\right) G_{c} G_{f f} e^{-T_{d} s}}{\Delta_{\mathrm{tol} 0}} .
$$

On the other hand, the transfer function of PI individual control is given by

$$
H_{\mathrm{PI}, d \rightarrow y}=\frac{G_{c} G_{f f} e^{-T_{d} s}}{\Delta_{\mathrm{PI}}} .
$$

Since the denominator of (22) and (23) is approximately the same, the only difference of these two transfer functions is the block $\left(1-Q G_{T_{d}}\right)$ which has a bode plot as shown in Fig. 8. According to its magnitude response, the factor $\left(1-Q G_{T_{d}}\right.$ ) will improve the system response (from $\mathrm{d}$ to $\mathrm{y}$ ) of the PI + DOB control in the frequency range of DC to $5 \mathrm{kHz}$.

Figure 7(b) depicts the system response from the noise signal, $n$, to cavity pickup, $y$. The DOB control has the best noise rejection among the three systems. There is no obvious difference between the PI control and the PI + DOB control. There may be some deterioration in the PI + DOB control, but it is minimal.

Figure 7(c) gives the system response from the reference signal, $r$, to the cavity pickup signal, $y$, corresponding to the transfer function from $r$ to $y$. This one is significant because the reference phase needs to be adjusted frequently during the beam commissioning. No obvious difference can be observed in the PI control and the PI + DOB control.

The system response from noise, $n$, to the DAC input, $\varepsilon$, is plotted in Fig. 7(d). The transfer function is investigated here owing to the restriction of if source. If the gain from $n$ to $\varepsilon$ is too large, input-power interlock occurs; however, there is no distinction after injecting the DOB controller into PI feedback loops.

\section{B. Robustness of DOB control}

To examine the robustness of the DOB control, we mainly consider two cases. In the first case, we suppose that the system is a SISO system, that is to say, the cavity is perfectly on resonance $(\delta \omega=0)$, and there is not any phase calibration error $(\delta \theta=0)$, however, there is mismatch in the parameters of the nominal model, $G_{n}(s)$. In the second case, we suppose that the detuning, $\delta \omega$, and the phase calibration error $\delta \theta$ (in Fig. 4) cannot be neglected and the plant $G_{p}(s)$ then becomes a MIMO system.

For the first case, because the two dominant parameters in $G_{n}(s)$ are the plant gain, $G_{f f}$, and the cavity bandwidth, $f_{0.5}$, we inspected them first. Note that here we still keep the models of $F(z), C_{P I}(z), Q(z)$ and $G_{f f} G_{c}(s)$ the same
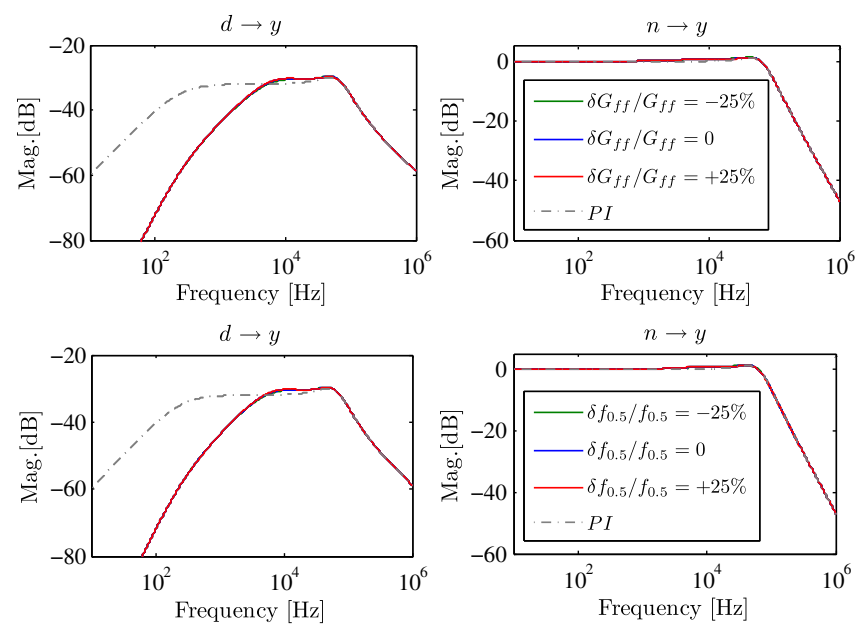

FIG. 9. Closed-loop transfer functions from $d$ and $n$ to $y$ in the $\mathrm{PI}+\mathrm{DOB}$ control (assuming $\delta \omega=0$ and $\delta \theta=0$ ). The figure shows the matched and mismatched cases, as well as the PI individual control case (indicated by the grey dash-dotted curve). From top to bottom: mismatch in plant gain $\left(G_{f f}\right)$, mismatch in cavity bandwidth $\left(f_{0.5}\right)$.

with Fig. 5. Equations (24) and (25) express the model mismatch in $G_{f f}$ and $f_{0.5}$, respectively. In this paper, we suppose that the mismatch for $G_{f f}$ and $f_{0.5}$ is less than $25 \%$, a restriction that is very easy to satisfy:

$$
\begin{aligned}
& G_{n}(s)=\left(G_{f f}+\delta G_{f f}\right) \frac{2 \pi f_{0.5}}{s+2 \pi f_{0.5}} . \\
& G_{n}(s)=G_{f f} \frac{2 \pi\left(f_{0.5}+\delta f_{0.5}\right)}{s+2 \pi\left(f_{0.5}+\delta f_{0.5}\right)} .
\end{aligned}
$$

Figure 9 shows the influence of the mismatch in plant gain, $G_{f f}$ (top), and cavity bandwidth, $f_{0.5}$ (bottom). We still consider the system response from $n$ and $d$ to $y$. According to Fig. 9, the mismatch in the two dominant
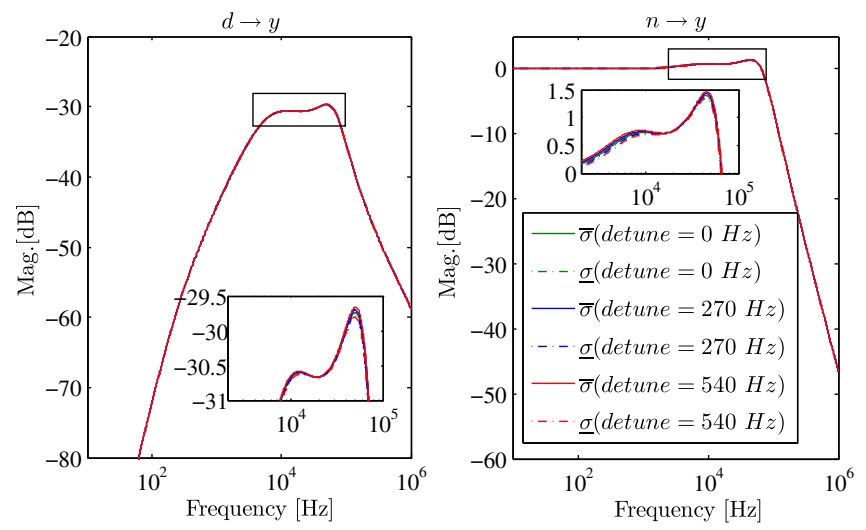

FIG. 10. The singular value of the closed-loop transfer function matrix from $d$ and $n$ to $y$ in terms of different cavity detuning $\delta \omega$. The $\bar{\sigma}$ in each transfer function indicates their $H_{\infty}$ norm. 

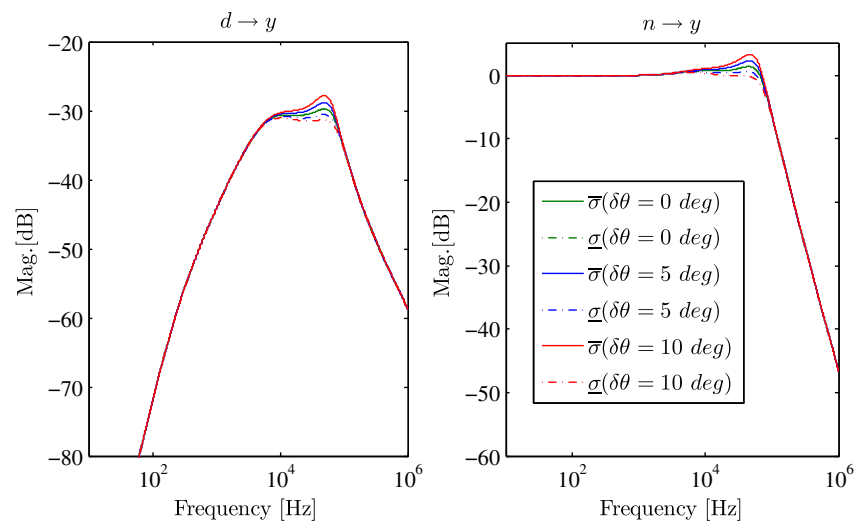

FIG. 11. The singular value of the closed-loop transfer function matrix from $d$ and $n$ to $y$ in terms of different phase calibration error $\delta \theta$ (see Fig. 4). The $\bar{\sigma}$ in each transfer function indicates their $H_{\infty}$ norm.

parameters does not influence the system performance severely.

For the second case, both the plant and the total LLRF system are MIMO systems. The $H_{\infty}$ norms of the closed loop system transfer function from $d$ and $n$ to $y$ are inspected.

Figures 10 and 11 illustrate the $H_{\infty}$ norm of these two transfer functions in terms of different detuning, $\delta \omega$, and phase calibration error, $\delta \theta$. Here, we exaggerate the cavity detuning to $540 \mathrm{~Hz}$ (same with the cavity half-bandwidth), in the real case, the detuning in injector 1 is controlled to be less than $50 \mathrm{~Hz}$ by piezo tuner. According to the analysis study of the $H_{\infty}$ norm, the phase calibration error, $\delta \theta$ will influence the system performance greater then the cavity detuning, $\delta \omega$.

\section{C. $Q$ filter and robust stabilities}

The selection of the $Q$ filter is a key point of the DOB control. To inspect the influence of the $Q$ filter, we consider $Q_{01}(s), Q_{02}(s)$, and $Q_{13}(s)$ in terms of (3) as shown in Table III.
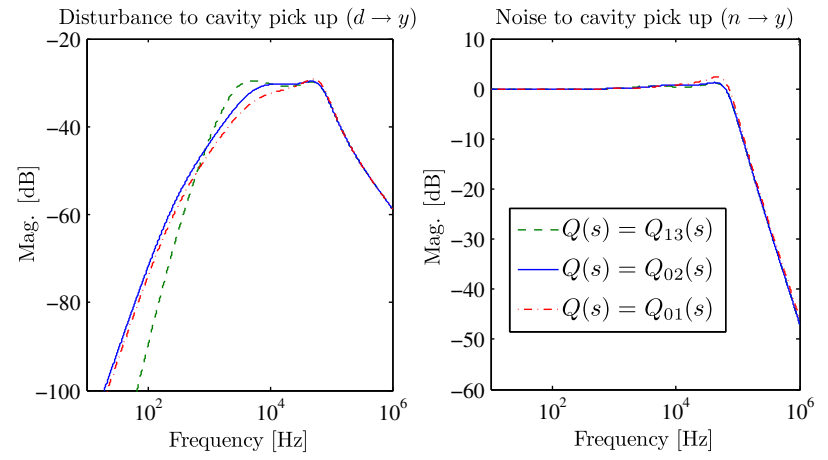

FIG. 12. Transfer functions from $d$ and $n$ to $y$ via different $Q$ filters in the PI + DOB control (assuming $\delta \omega=0$ and $\delta \theta=0$ ).
Figure 12 shows the transfer function from $d$ and $n$ to $y$ according to different $Q$ filters but with the same bandwidth (5 kHz). The filter $Q_{13}(s)$ is the most attractive filter as it provides the best performance in terms of disturbance rejection for the frequencies in the range of DC to $800 \mathrm{~Hz}$. The filter $Q_{01}(s)$ showed the poorest performance for noise suppression. However, in terms of the FPGA implementation, $Q_{13}$ will consume the most hardware resources. In order to find a balance between controller complexity and performance, we finally selected $Q_{02}(s)$ for the DOB control (in both the analytical study in this paper and the applications during the cERL commissioning).

The effect of the $Q_{02}$ filter on the cutoff bandwidth ( $3 \mathrm{~dB}$ bandwidth), $f_{Q}$, is shown in Fig. 13. Figures 13(a) and 13(b) show the performance of the PI + DOB control and "FF + DOB" control (no PI feedback), respectively. The selection of $f_{Q}$ is a trade-off between disturbance rejection and noise suppression according to Fig. 13. The $f_{Q}$ of the $\mathrm{PI}+\mathrm{DOB}$ control is selected to be $5 \mathrm{kHz}$ to reach a compromise.

Finally in the end of the analytical study, we would like to present the analysis of the robust stability. The real LLRF system $G_{p}(s)$ is subjected to multiplicative model uncertainties (e.g., cavity is detuned by some reason), which can be described by [10,15-17]

$$
G_{p}(s)=G_{n}(s)[1+\Delta(s)]
$$

where $\Delta(s)$ is the variable transfer function, which describes uncertainties of the plant. The robust stability of the DOB controller is then assured by $[10,15-17]$
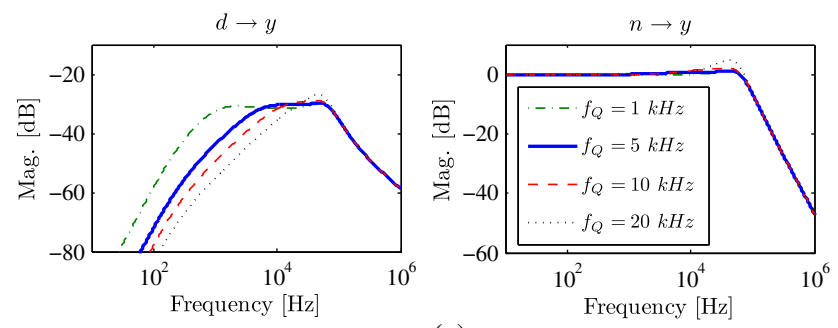

(a)
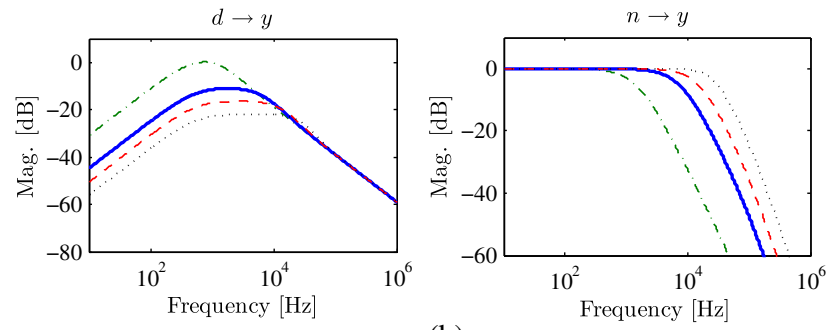

(b)

FIG. 13. Transfer functions from $d$ and $n$ to $y$ via different $Q$ filter bandwidths (assuming $\delta \omega=0$ and $\delta \theta=0$ ). (a) PI $+\mathrm{DOB}$ control and (b) FF + DOB control. 


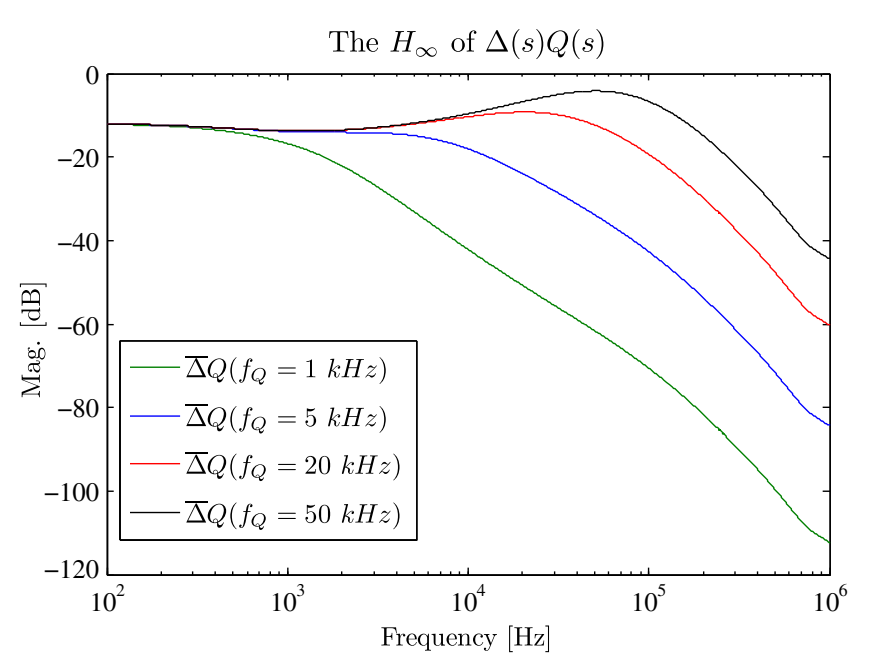

FIG. 14. The $H_{\infty}$ norm of $\Delta(s) Q(s)$. Assuming the detuning is $50 \mathrm{~Hz}$ and the phase calibration error is 10 degrees in the system. In addition, there is no mismatch in $G_{f f}$ and $f_{0.5}$.

$$
\|\left(\Delta(j \omega) Q(j \omega) \|_{\infty} \leq 1,\right.
$$

Figure 14 plots the $H_{\infty}$ of (14) in terms of different $Q_{02}$ filter bandwidth, $f_{Q}$. Here we assume the detuning of the cavity, $\delta \omega$ is $50 \mathrm{~Hz}$ and the phase calibration error, $\delta \theta$, is 10 degrees. These restrictions are very easy to satisfy in the cERL. It is clear to see from Fig. 14 that if the bandwidth of the $Q$ filter is larger than $50 \mathrm{kHz}$, the DOB control is subject to a risk of unstable. In the experiment during the beam commissioning, we have limited the $Q$ filter bandwidth, $f_{Q}$ to be less than $25 \mathrm{kHz}$.

\section{EXPERIMENT ON CERL BEAM COMMISSIONING}

To validate the DOB control in an actual accelerator, we installed this controller in the LLRF system for both injectors and the main linac. The main parameters of the LLRF systems at cERL are given in Tables IV and V, respectively. Note that for the cavities in injector 2 and injector 3, the vector-sum control is applied; in this case, the combination system is, at least, a second-order model.

TABLE IV. LLRF and cavity parameters of injectors in the cERL.

\begin{tabular}{lccc}
\hline \hline Cavity & Injector1 & Injector2 & Injector3 \\
\hline Cavity voltage $\left(V_{c}\right)$ & $0.7 \mathrm{MV}$ & $0.65 \mathrm{MV}$ & $0.65 \mathrm{MV}$ \\
Beam phase $\left(\phi_{b}\right)$ & $0^{\circ}$ & $0^{\circ}$ & $0^{\circ}$ \\
Control method & Individual control & Vector-sum control \\
Power source & $20 \mathrm{~kW}$ klystron & $300 \mathrm{~kW}$ & klystron \\
Loaded Q $\left(Q_{L}\right)$ & $1.2 \times 10^{6}$ & $5.7 \times 10^{5}$ & $4.8 \times 10^{5}$ \\
Bandwidth $\left(f_{0.5}\right)$ & $540 \mathrm{~Hz}$ & $1120 \mathrm{~Hz}$ & $1350 \mathrm{~Hz}$ \\
Time delay $\left(T_{n}\right)$ & $1 \mu \mathrm{s}$ & $1 \mu \mathrm{s}$ & $1 \mu \mathrm{s}$ \\
FF gain $\left(G_{f f}\right)$ & 2.13 & 0.69 (vector-sum) \\
\hline \hline
\end{tabular}

TABLE V. LLRF and cavity parameters of the main linac in the cERL.

\begin{tabular}{lcc}
\hline \hline Cavity & Main linac1 & Main linac2 \\
\hline Cavity voltage $\left(V_{c}\right)$ & $8.5 \mathrm{MV}$ & $8.5 \mathrm{MV}$ \\
Beam phase $\left(\phi_{b}\right)$ & $0^{\circ}$ & $0^{\circ}$ \\
Control method & Individual control & Individual control \\
Power source & $16 \mathrm{~kW} \mathrm{SSA}$ & $8 \mathrm{~kW} \mathrm{SSA}$ \\
Loaded Q $\left(Q_{L}\right)$ & $1.3 \times 10^{7}$ & $1.0 \times 10^{7}$ \\
Bandwidth $\left(f_{0.5}\right)$ & $50 \mathrm{~Hz}$ & $65 \mathrm{~Hz}$ \\
Time delay $\left(T_{n}\right)$ & $1 \mu \mathrm{s}$ & $1 \mu \mathrm{s}$ \\
FF gain $\left(G_{f f}\right)$ & 1.36 & 1.14 \\
\hline \hline
\end{tabular}

Because of the robustness of DOB control, we applied a first-order model such as (7) as the nominal model and selected the cavity half-bandwidth to be $1300 \mathrm{~Hz}$ to represent the overall $f_{0.5}$.

System performance was improved greatly after applying the DOB control. Because the motivation for use of the DOB control was to reject disturbance signals, we will discuss the improvement in terms of different disturbances.

\section{A. Power-supply ripples rejection}

The power supply of the $300 \mathrm{~kW}$ klystron for injectors 2 and 3 is disturbed by $300 \mathrm{~Hz}$ ripples, as shown in Fig. 15 . These $300 \mathrm{~Hz}$ ripples can be suppressed by applying high FB gains. However, large gains not only increase the risk of an unstable system but also deteriorate the high-frequency noise level. The optimum FB gain is a trade-off between disturbance rejection and system stability. The current FB gain was optimized by a gain-scanning experiment $[24,26]$. However, during beam commissioning, the optimal gain could not remove the $300 \mathrm{~Hz}$ power-supply ripples completely.

The present DOB control, combined with the original PI control, was applied to reject the power ripples. At first, we tried to operate the LLRF system with FF control; however, owing to the effects of the power-supply ripples, the amplitude and phase were seriously disturbed. The blue curves in Fig. 16(a) give the amplitude and phase performance and their corresponding fast Fourier transform

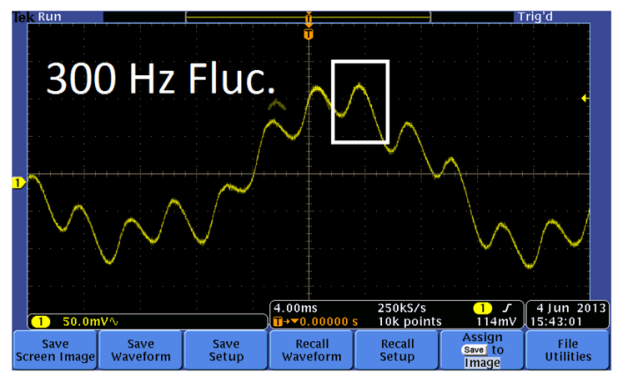

FIG. 15. $300 \mathrm{~Hz}$ fluctuation in the power-supply output measured by the oscilloscope. 

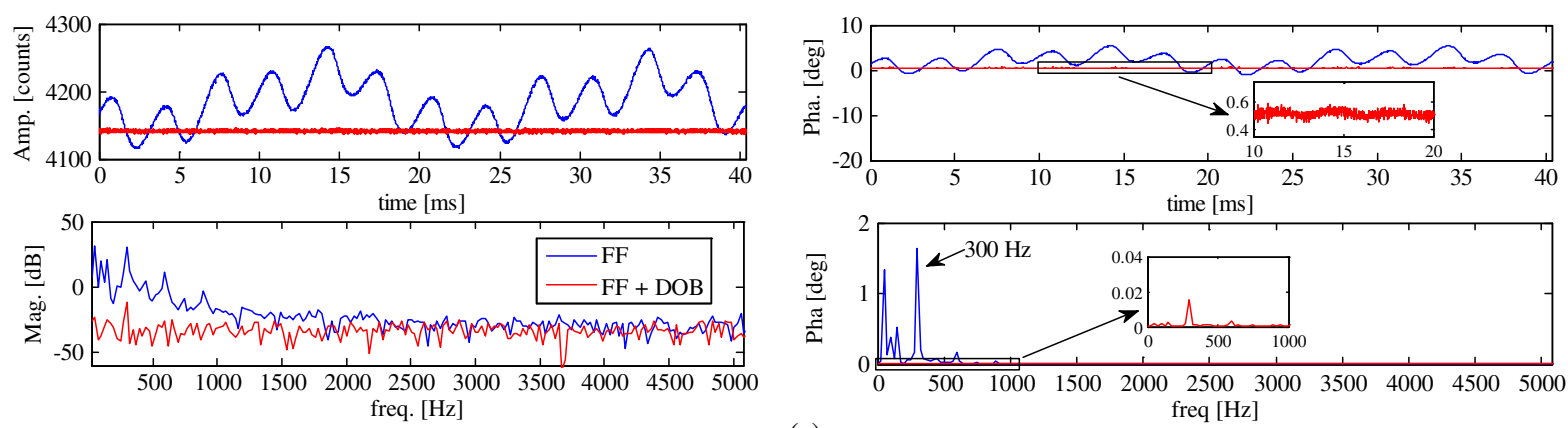

(a)
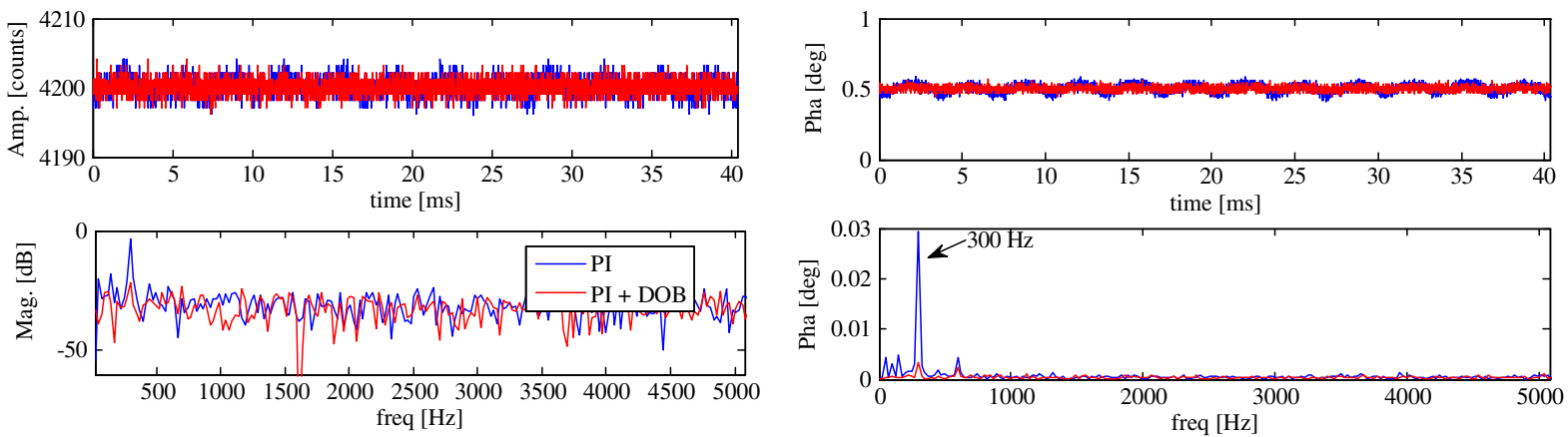

(b)

FIG. 16. Original FF/FB control (blue) vs FF/FB + DOB control (red). Radio-frequency field performance in amplitude and phase is plotted, as well as their corresponding FFT analyses. (a) Under the operation of FF control $\left(f_{Q}=20 \mathrm{kHz}\right)$, the blue and red curves indicate DOB off and on, respectively. (b) Under the operation of FB control ( $\left.f_{Q}=2 \mathrm{kHz}\right)$, the blue and red curves correspond to the PI feedback control and PI + DOB control, respectively.

(FFT) analysis under FF operation. The effects of the $300 \mathrm{~Hz}$ power ripples are apparent. We applied the DOB control (without PI feedback) with FF operation [indicated by the red waveform in Fig. 16(a)], and the $300 \mathrm{~Hz}$ component suppression greatly improved.

In the next step, we switched off DOB control and operated the LLRF system with PI feedback. The $300 \mathrm{~Hz}$ ripples were still rejected, but they could still be observed in the measured rf field, especially on the waveform of the phase [indicated by blue waveforms in the right subplot of Fig. 16(b)]. The system performance improved greatly after applying the DOB control together with PI feedback. The red curve in Fig. 16(b) indicates that the $300 \mathrm{~Hz}$ ripples almost disappeared.

It should be noted that the selection of the $Q$ filter bandwidth $f_{Q}$ in DOB control for the FF and FB operations is recommended to be different according to our experience. For a better performance, the $f_{Q}$ in the FF operation should be larger than that in the FB operation. This point was also demonstrated in the analytical study, as shown in Fig. 13. In the measurement shown in Fig. 16, the values of $f_{Q}$ were selected to be 20 and $2 \mathrm{kHz}$ in the FF and FB operations, respectively.

Table VI summarizes the improvement of DOB control in comparison to the original PI control in both FF and FB operations. The influence of the $Q$ filter is presented as well.

\section{B. Beam-loading compensation}

Beam-loading compensation is one of the key issues in accelerators. To demonstrate the DOB control in the present beam-loading case, an LLRF experiment with a beam was performed in the cERL operated under burst mode $(5 \mathrm{~Hz})$. As a comparison, both PI control and PI + DOB control were applied in sequence, and the FB gain in the PI controller was set to the optimal value. Since all of the cavities in the injector were operated in the on-crest mode

TABLE VI. Improvement of DOB control on FF and FB operation.

\begin{tabular}{|c|c|c|c|c|}
\hline \multirow{2}{*}{\multicolumn{2}{|c|}{ Control method }} & & \multicolumn{2}{|c|}{ rf field stability } \\
\hline & & & $\Delta A / A[\% \mathrm{rms}]$ & $\Delta \theta\left[^{\circ} \mathrm{rms}\right.$ \\
\hline \multirow{4}{*}{ FF } & \multicolumn{2}{|c|}{ DOB off } & $0.854 \%$ & $1.591^{\circ}$ \\
\hline & \multirow{3}{*}{ DOB on } & $f_{Q}=5 \mathrm{kHz}$ & $0.038 \%$ & $0.051^{\circ}$ \\
\hline & & $\overline{f_{Q}=10 \mathrm{kHz}}$ & $0.030 \%$ & $0.030^{\circ}$ \\
\hline & & $f_{Q}=20 \mathrm{kHz}$ & $0.030 \%$ & $0.023^{\circ}$ \\
\hline \multirow{4}{*}{ FB } & \multicolumn{2}{|c|}{ DOB off } & $0.030 \%$ & $0.029^{\circ}$ \\
\hline & \multirow{3}{*}{ DOB on } & $f_{Q}=1 \mathrm{kHz}$ & $0.026 \%$ & $0.018^{\circ}$ \\
\hline & & $f_{Q}=2 \mathrm{kHz}$ & $0.026 \%$ & $0.017^{\circ}$ \\
\hline & & $\overline{f_{Q}=5 \mathrm{kHz}}$ & $0.026 \%$ & $0.018^{\circ}$ \\
\hline
\end{tabular}




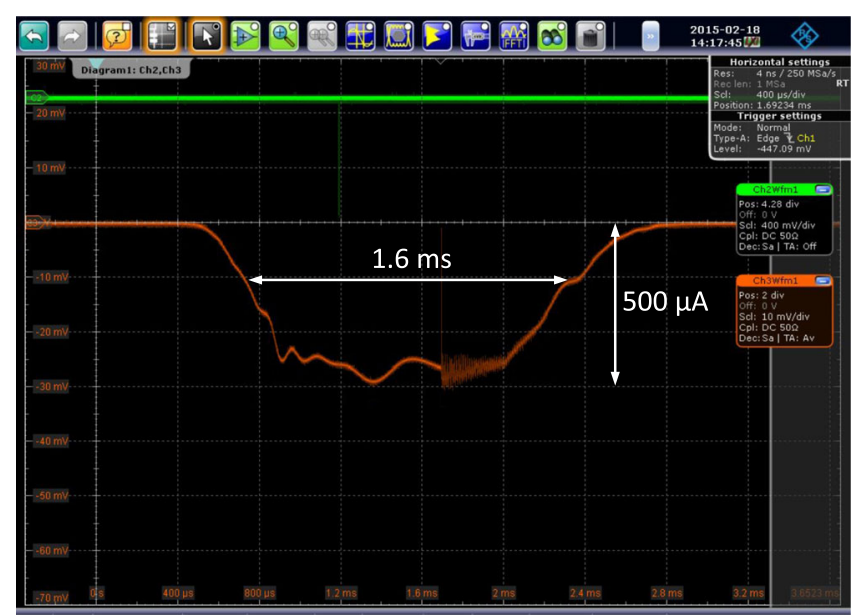

FIG. 17. The $1.6 \mathrm{~ms}$ and $500 \mu \mathrm{A}$ beam current measured by oscilloscope.

(see Table IV), the beam-loading effects were concentrated mainly in the amplitude of the rf field.

The pulse width of the beam was selected to be approximately $1.6 \rightarrow 2.0 \mathrm{~ms}$, and we increased the beam current from 140 to 500 and $800 \mu \mathrm{A}$ (see Fig. 17). In the case of the $140 \mu \mathrm{A}$ beam, both PI control and PI + DOB worked well for beam compensation, and the $300 \mathrm{~Hz}$ PS ripples were well rejected by the PI + DOB control.

However, after increasing the beam current up to 500 and $800 \mu \mathrm{A}$, the beam effects were obvious in the rf field, especially for the rf amplitude. Figure 18 shows the measured rf field with $800 \mu \mathrm{A}$ beam loading in the cavities of injectors 1-3. In each subplot, the blue and red waveforms indicate the measured rf field under PI control and PI + DOB control, respectively. It is clear that the current PI control cannot provide sufficient compensation for high-intensity beam loading. Experiments under 500 and
$800 \mu \mathrm{A}$ beam loading demonstrated that the beam-loading effects were greatly improved by the DOB controller.

Table VII gives the measured rf field stability in terms of different beam currents and control methods. The improvement is obvious for the PI + DOB control, especially in the case of high-intensity beam currents.

The bandwidth $f_{Q}$ of the $Q$ filter was increased to $5 \mathrm{kHz}$ in the PI + DOB control (as compared to $2 \mathrm{kHz}$ for the power-supply ripples suppression). The main reason is that the spectrum in the beam loading contains frequency components higher than $300 \mathrm{~Hz}$. Therefore, we need a $Q$ filter with a larger bandwidth to cover the high-frequency band in the beam pulse.

\section{Microphonics rejection}

Two nine-cell cavities (main linac 1 and main linac 2) were employed in the main linac at cERL. The nine-cell cavities are subjected to the disturbances of microphonics. In principle, these microphonics can be well rejected by applying high FB gains (e.g., less than 8 mdeg for both cavities), however, the microphonics still can be observed in the phase of the pickup signal. Especially, in the cavity of main linac 2 , there is a $49.6 \mathrm{~Hz}$ dominant component in the measured pickup signal.

To further improve the performance of the microphonics rejection, we have applied the DOB control in the LLRF systems. Figure 19 compares the PI + DOB control (red) and PI individual control (blue), and the improvement is obvious due to the microphonics is almost disappeared after switching on the DOB switch.

\section{Experience and discussion}

Based on the successful attempt of DOB control in the cERL LLRF system during beam commissioning, we
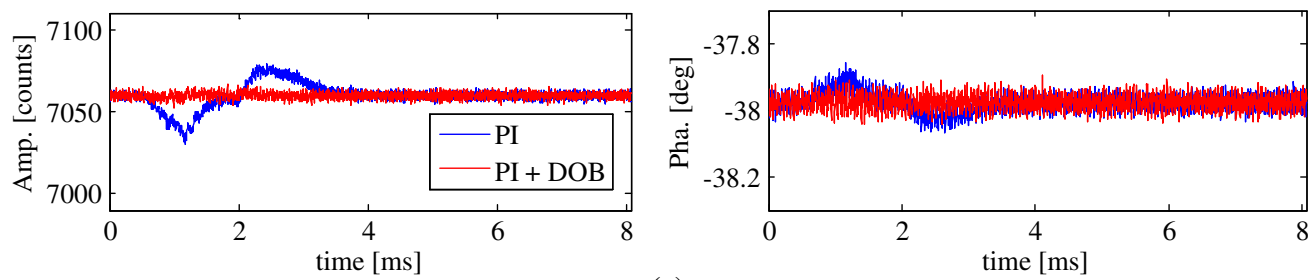

(a)
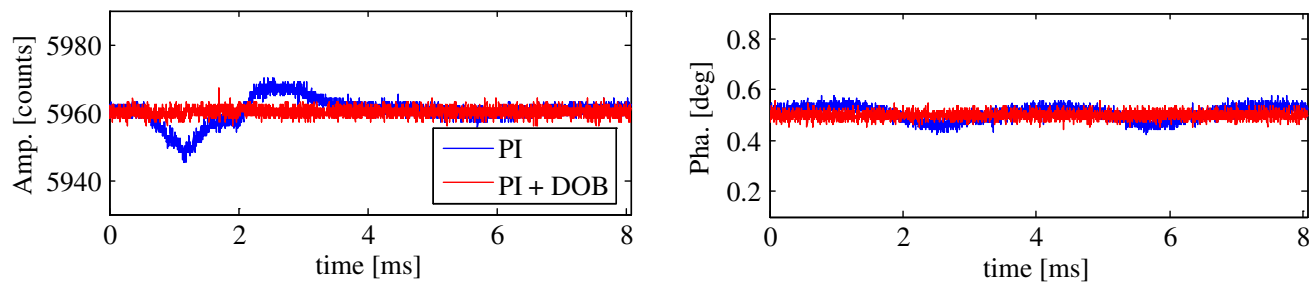

(b)

FIG. 18. The measured rf field for the amplitude (left) and the phase (right) on the cavities of the injectors in the presence of $800 \mu \mathrm{A}$ beam loading. The blue and red waveforms in each subplot represent the PI control and PI + DOB control $\left(f_{Q}=5 \mathrm{kHz}\right)$, respectively. The operated beam currents are (a) injector 1 and (b) injectors 2 and 3. 
TABLE VII. Performance of DOB control in the beam-loading compensation during the cERL beam commissioning.

\begin{tabular}{|c|c|c|c|c|}
\hline \multirow[b]{2}{*}{ Cavity } & \multirow[b]{2}{*}{$I_{b}$} & \multirow[b]{2}{*}{ Method } & \multicolumn{2}{|c|}{ rf field stability } \\
\hline & & & $\Delta A / A[\% \mathrm{rms}]$ & $\Delta \theta\left[^{\circ} \mathrm{rms}\right]$ \\
\hline \multirow{6}{*}{ Injector 1} & \multirow{2}{*}{$140 \mu \mathrm{A}$} & PI & $0.027 \%$ & $0.018^{\circ}$ \\
\hline & & $\mathrm{PI}+\mathrm{DOB}$ & $0.024 \%$ & $0.021^{\circ}$ \\
\hline & \multirow{2}{*}{$500 \mu \mathrm{A}$} & PI & $0.060 \%$ & $0.021^{\circ}$ \\
\hline & & $\overline{\mathrm{PI}+\mathrm{DOB}}$ & $0.026 \%$ & $0.021^{\circ}$ \\
\hline & \multirow{2}{*}{$800 \mu \mathrm{A}$} & PI & $0.100 \%$ & $0.027^{\circ}$ \\
\hline & & $\mathrm{PI}+\mathrm{DOB}$ & $0.026 \%$ & $0.022^{\circ}$ \\
\hline \multirow{6}{*}{$\begin{array}{l}\text { Injectors } 2 \text { and } 3 \\
\text { Vector sum }\end{array}$} & \multirow{2}{*}{$140 \mu \mathrm{A}$} & PI & $0.026 \%$ & $0.026^{\circ}$ \\
\hline & & $\mathrm{PI}+\mathrm{DOB}$ & $0.021 \%$ & $0.016^{\circ}$ \\
\hline & \multirow{2}{*}{$500 \mu \mathrm{A}$} & PI & $0.051 \%$ & $0.025^{\circ}$ \\
\hline & & $\overline{\mathrm{PI}+\mathrm{DOB}}$ & $0.021 \%$ & $0.016^{\circ}$ \\
\hline & \multirow{2}{*}{$800 \mu \mathrm{A}$} & PI & $0.063 \%$ & $0.027^{\circ}$ \\
\hline & & $\mathrm{PI}+\mathrm{DOB}$ & $0.021 \%$ & $0.016^{\circ}$ \\
\hline
\end{tabular}

would like to summarize our experience as follows: (i) A mathematical LLRF system model is required, but a rough model is good enough because of the robustness of the DOB controller. As presented above, what we have implemented in the present DOB controller is only a first-order cavity model with only two parameters: plant gain, $G_{f f}$, and cavity bandwidth, $f_{0.5}$ (or loaded Q value). The identification of these parameters is rather easy and convenient. (ii) In comparison to identification of the parameters in the nominal cavity model $\left(G_{f f}\right.$, and $\left.f_{0.5}\right)$ and the nominal time delay $\left(T_{n}\right)$ the determination of the $Q$ filter is much more significant. Both the filter type and the filter bandwidth will influence the performance of the DOB control. Here, we selected $Q(s)=Q_{02}(s)$ because of the limitation of FPGA hardware resources, but other selections could be even better according to the analytical study. The filter cutoff bandwidth, $f_{0.5}$, should be selected mainly in terms of the frequency band of the interested disturbance signal. (iii) In principle, the combination of DOB controller will not influence the original PI loops if the rf plant is identified perfectly (no mismatch, no detuning and no phase calibration error); that is, characteristics of the original loop such as gain margin, phase margin, critical gains and closed bandwidth are almost the same whether switching the DOB controller on or off (see Figs. 6 and 7). In practice, these characteristics mentioned above (gain margin, phase margin, critical gains and closed bandwidth) are influenced owing to the mismatch model and the model uncertainties $(\delta \omega \neq 0$ and/or $\delta \theta \neq 0)$, but the influence is acceptable according to our experience in the cERL beam commissioning.

The possible limitations of DOB control are discussed as follows: (i) The effective bandwidth of DOB control is limited by the $Q$ filter (which determines the effective bandwidth observed by the inverse plant model). Since the bandwidth of the $Q$ filter, $f_{Q}$, is limited less than $50 \mathrm{kHz}$ by the robust stability requirements (see Fig. 14), therefore, if the frequency of the disturbance signal is larger than 50 $\mathrm{kHz}$ (e.g., $1 \mu$ s beam loading in the burst mode), the DOB control does not work. On the other hand, if the frequency of the disturbance signal is concentrated on a very low frequency (DC to $10 \mathrm{~Hz}$ ), the DOB control works, but there
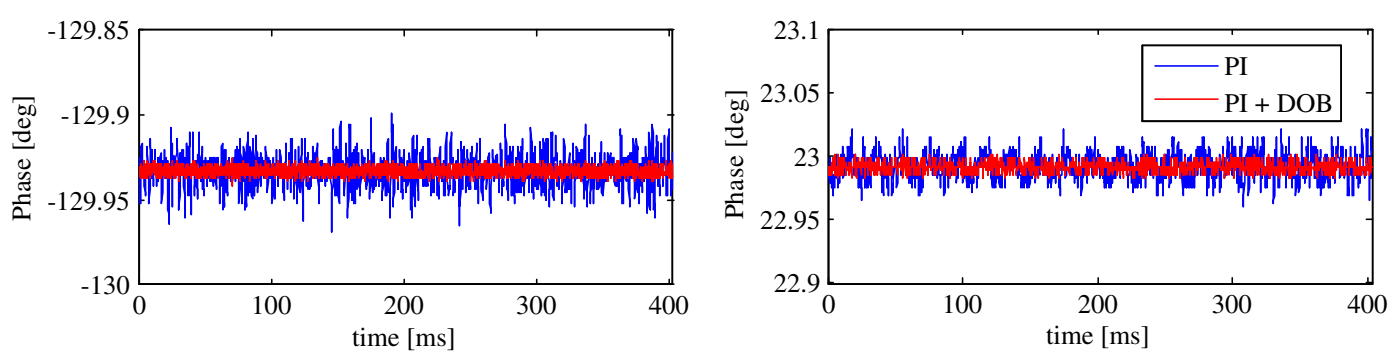

(a)
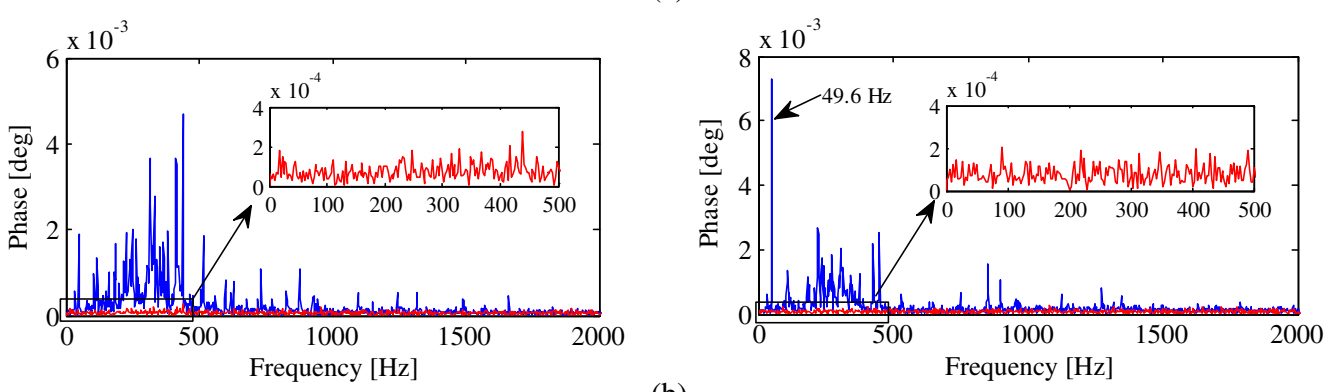

(b)

FIG. 19. Measured rf phase of the pickup signal in the main linac1 (left) and main linac2 (right) cavities in the case of PI individual control (blue) and PI + DOB control (red) with $5 \mathrm{kHz} Q$ filter. From top to bottom: (a) waveform, (b) spectrum. 
are no obvious advantages to using DOB control as compared with traditional PI control. (ii) The DOB control is very robust, and even a first-order model worked well. However, if the system is very seriously detuned (e.g., large Lorentz detuning), the controller may not work. In this case, we may need modern system identification for a complex system model that includes high-order dynamic behavior.

\section{SUMMARY}

Disturbances such as microphonics, power-supply ripples, and beam loading often exist in LLRF systems. The traditional PI control is not sufficient to reject these disturbance signals. To solve this problem, an application of a disturbance observer-based control for SCRF cavity regulation is presented in this paper. The basic idea of the controller is that we estimate the disturbance signal by applying the inverse system model, and we remove the estimated disturbance in the FF models.

This DOB controller was analyzed with Matlab, and then developed and implemented in the FPGA-based digital LLRF system in the cERL. In the beam commissioning, the validation of this controller was demonstrated by successfully compensating the high-intensity beam loading in burst mode, as well as by successfully rejecting the $300 \mathrm{~Hz}$ HVPS ripples and microphonics. This controller is now installed in the LLRF system of cERL at KEK under normal operation.

[1] H. Abe et al., Energy recovery linac preliminary design report, Design report of 3-GeV ERL, Japan.

[2] S. Sakanaka et al., Construction and commissioning of compact-ERL injector at KEK, in Proceedings of the 53th ICFA Advanced Beam Dynamics Workshop on Energy Recovery Linacs, ERL-2013, Novosibirsk, Russia, 2013 (JACoW, Novosibirsk, Russia, 2013), WG102, p. 16.

[3] N. Nakamura et al., Present status of the compact ERL at KEK, in Proceedings of the fifth International Particle Accelerator Conference, IPAC'14, Dresden, Germany, 2014 (JACoW, Dresden, Germany, 2014), MOPRO110, p. 353.

[4] K. Watanabe, S. Noguchi, E. Kako, K. Umemori, and T. Shishido, Development of the superconducting of 2-cell cavity for cERL Injector at KEK, Nucl. Instrum. Methods Phys. Res., Sect. A 714, 67 (2013).

[5] K. Umemori et al., Operation status of compact ERL main linac crymodules, in Proceedings of the fifth International Particle Accelerator Conference, IPAC'14, Dresden, Germany, 2014 (JACoW, Dresden, Germany, 2014), WEPRI028, p. 2537.

[6] T. Miura et al., Performance of rf systems for compactERL Injector at KEK, in Proceedings of the 53th ICFA Advanced Beam Dynamics Workshop on Energy Recovery Linacs, ERL-2013, Novosibirsk, Russia, 2013 (JACoW, Novosibirsk, Russia, 2013), WG306, p. 58.
[7] M. Satoh et al., Mechanical vibration search of compact ERL main linac superconducting cavities in cryomodule, in Proceedings of the 5th International Particle Accelerator Conference, IPAC'14, Dresden, Germany, 2014 (JACoW, Dresden, Germany, 2014), WEPRI026, p. 2531.

[8] T. Miyajima et al., Process in construction of gun test facility for compact ERL, in Proceedings of the first International Particle Accelerator Conference, IPAC'10, Kyoto, Japan, 2010 (OC/ACFA, Kyoto, Japan, 2010), TUPE090, p. 2335.

[9] K. Ohishi, Microprocessor controlled dc motor for load insensitive position servo system, IEEE Transact. Indust. Electron. IE-34, 44 (1987).

[10] X. Chen and M. Tomizuka, Lecture notes for UC Berkeley Advanced Control Systems II (ME233), available at http:// www.me.berkeley.edu/ME233/sp14, 2014.

[11] T. Umeno and Y. Hori, Robust speed control of dc servomotors using modern two degrees-of-freedom controller design, IEEE Trans. Indust. Electron. 38, 363 (1991).

[12] Y. Choi, K. Yang, W. K. Chung, H. R. Kim, and I. H. Suh, On the robustness and performance of disturbance observers for second-order systems, IEEE Trans. Autom. Control 48, 315 (2003).

[13] S. Wang, C. H. Lu, and A.C. Lee, Disturbance observer structure applied to sensorless brushless DC motors drive, Int. J. Comp. Theory Eng. 7, 92 (2015).

[14] R. H. Horng, H.-L. Chou, and A.-C. Lee, Rejection of limit cycles induced from disturbance observers in motion control, IEEE Trans. Indust. Electron. 53, 1770 (2006).

[15] C. J. Kempf et al., Discrete-time disturbance observer design for systems with time delay, in Proceedings of the 4th International Workshop on Advanced Motion Control (AMC'96-MIE) (IEEE, New York, 1996), Vol. 1, pp. 332-337.

[16] X.Chen et al., Optimal plant shaping for high bandwidth disturbance rejection in discrete disturbance observers, in the American Control Conference (ACC) (IEEE, New York, 2010), pp. 2641-2646.

[17] W. Li and Y. Hori, Vibration suppression using single neuron-based PI fuzzy controller and fractional-order disturbance observer, IEEE Trans. Indust. Electron. 54, 117 (2007).

[18] J.E. Normey-Rico and E. F. Camacho, Control of Dead-time Processes (Springer, London, 2007), Chap. 6.

[19] C. J. Kempf and S. Kobayashi, Disturbance observer and feedforward design for a high-speed direct-drive positioning table, IEEE Trans. Control Syst. Technol. 7, 513 (1999).

[20] Y.I. Son, G. J. Jeong, and I. H. Kim, Disturbance observer based position control of a one-link manipulator under input time delay, Int. J. Innov. Comput. Inf. Control 8, 1371 (2012).

[21] F. Qiu et al., A disturbance-observer-based controller for LLRF systems, in Proceedings of the sixth International Particle Accelerator Conference, IPAC'15, Richmond, USA, 2015 (JACoW, Richmond, USA, 2015), WEPMA054, p. 2895.

[22] T. Matsumoto et al., Digital low-level rf control system with four intermediate frequencies at STF, in 
Proceedings of the 23rd Particle Accelerator Conference, Vancouver, Canada, 2009 (JACoW, Vancouver, Canada, 2009), WE5PFP086, p. 2198.

[23] T. Schilcher, RF application in digital signal processing, in Proceedings of CERN Accelerator School on Digital Signal Processing, CAS2007, Sigtuna, Sweden, 2007 (CERN, Geneva, Switzerland, 2008), p. 249.

[24] F. Qiu et al., Evaluation of the superconducting LLRF system at cERL in KEK, in Proceedings of the 4th International Particle Accelerator Conference, IPAC2013, Shanghai, China, 2013 (JACoW, Shanghai, China, 2013).

[25] F. Qiu et al., Digital filters used for digital feedback system at cERL, in Proceedings of the 27th Linear Accelerator Conference, LINAC14, Geneva, Switzerland, 2014 (JACoW, Geneva, Switzerland, 2014), MOPP074, p. 227.

[26] F. Qiu, Performance of cw superconducting cavity at ERL test facility, in Oral presentation of the sixth Low-Level
Radio Frequency Workshop, LLRF13, Lake Tahoe, USA, 2013 (LBNL, Lake Tahoe, USA, 2013).

[27] F. Qiu et al., Performance of the digital LLRF system at the cERL, in Proceedings of the fifth International Particle Accelerator Conference, IPAC'14, Dresden, Germany, 2014 (JACoW, Dresden, Germany, 2014), WEPME072, p. 2447.

[28] C. Schmidt, Ph.D. thesis, Technische Universität Hamburg-Harburg, 2010.

[29] T. Miura et al., Performance of rf systems for compact-ERL main linac at KEK, in Proceedings of the fifth International Particle Accelerator Conference, IPAC'14, Dresden, Germany, 2014 (JACoW, Dresden, Germany, 2014), WEPME073, p. 2450.

[30] T. Schilcher, Ph.D. thesis, Universität Hamburg, 1998.

[31] S. J. Mason, Feedback theory: Further properties of signal flow graphs, in Proceedings of the Institute of Radio Engineers (IRE, New York, 1956), pp. 920-926. 